\title{
Avaliação da Aplicabilidade de Técnicas FT-IR de Reflexão (UATR) e de Transmissão para a Determinação do Teor de Acrilonitrila (AN) em NBR
}

\author{
Natália B. Sanches \\ Instituto Tecnológico da Aeronáutica e Centro Logístico da Aeronáutica \\ Milton F. Diniz, Luis C. Alves, Jorge C. N. Dutra, Silvana N. Cassu, Margarete F. P. Azevedo, Rita C. L. Dutra \\ Divisão de Química, Instituto de Aeronáutica e Espaço, CTA
}

\begin{abstract}
Resumo: Amostras vulcanizadas de copolímero de butadieno e acrilonitrila (NBR), contendo teor conhecido de acrilonitrila (AN), foram preparadas nos laboratórios do IAE como amostras de referência para a elaboração de curvas analíticas (ou de calibração), visando à determinação do teor de AN em NBR, por meio de técnicas FT-IR de reflexão (UATR) de última geração e as conhecidas de transmissão. A preparação das amostras foi feita por pirólise, sem e com controle de temperatura, introduzindo uma nova forma de recolhimento do pirolisado. A banda FT-IR analítica, característica de AN, usada no estudo foi a encontrada em $2237 \mathrm{~cm}^{-1}$, sendo $1455 \mathrm{~cm}^{-1}$ a banda de referência, atribuída aos grupos $\mathrm{CH}_{2}$ e $\mathrm{CH}_{3}$. Os valores da absorvância relativa $\mathrm{A}_{2237} / \mathrm{A}_{1455} v s$. teores conhecidos (nominais) de $\mathrm{AN}$ em NBR foram usados para elaborar curvas analíticas com boas correlações lineares $(\mathrm{R}=0,995$ para técnica de transmissão/pirólise controlada e $\mathrm{R}=0,989$ para técnica de transmissão/pirólise em bico de Bunsen). Na técnica UATR, valores de $\mathrm{A}_{2237} v s$. os teores nominais de AN em NBR foram usados para elaborar uma curva analítica, que também apresentou boa correlação linear $(R=0,996)$. Ambas as curvas analíticas possibilitam a determinação dos teores de AN em NBR, em amostras em que os componentes têm a mesma composição química. Embora as técnicas apresentem precisão similar, o tempo para obtenção dos resultados (experimental e cálculo) é bem menor utilizando-se a técnica UATR para esse tipo determinação, especialmente quando o tempo de extração, etapa usada na técnica de transmissão/pirólise, é considerado.
\end{abstract}

Palavras-chave: Acrilonitrila, copolímero de butadieno e acrilonitrila ou borracha nitrílica, FT-IR, técnicas de transmissão, técnica de reflexão.

\section{Evaluation of FT-IR (UATR and Transmission) Techniques for Determination of AN Content in NBR Samples}

Abstract: NBR samples with known contents of acrilonitrile (AN) were prepared at IAE laboratories as reference samples for producing an analytical curve to determine the content of AN and NBR, using new methods of transmission (pyrolysis) and UATR/FT-IR techniques. The chosen FT-IR analytical band for AN was $2237 \mathrm{~cm}^{-1}$ for transmission (pyrolysis) and UATR technique. The reference band chosen in transmission pyrolysis technique was $1455 \mathrm{~cm}^{-1}$. Values of $\mathrm{A}_{2237} / \mathrm{A}_{1455}$ relative absorbance (transmission pyrolysis technique) and of $\mathrm{A}_{2237}$ (UATR technique) vs. the AN contents data were used to elaborate analytical curves with good linear correlations $(\mathrm{R}=0.995$ e $\mathrm{R}=0.989)$ - transmission pyrolysis technique $)$ and $\mathrm{R}=0.996$ (UATR technique), which allows one to determine contents of AN in NBR samples of similar composition. Even though the techniques showed similar accuracy, the time required for the analysis is smaller with the UATR technique, especially if the time for extraction for applying the transmission pyrolysis technique is also considered.

Keywords: Acrilonitrile, butadiene-acrilonitrile copolymer, FT-IR, transmission pyrolysis, UATR.

\section{Introdução}

Como é conhecido, propriedades de NBR, como tensão de ruptura, dureza, entre outras, variam em função do teor de $\mathrm{AN}^{[1,2]}$ Os teores de AN variam de $28 \%$ (baixo teor) a $45 \%$ (ultra-alto teor), existindo quatro teores básicos disponíveis, $18,33,39$ e $45 \%$, porém valores intermediários podem ser obtidos, misturando-se duas borrachas nitrílicas com teores diferentes de AN. As borrachas NBR de ultra-alto teor de AN (45\%) são empregadas na confecção de artefatos que trabalham sob condições extremas de resistência a óleos e uma ótima flexibilidade a baixas temperaturas. Em geral, as borrachas NBR de médio teor (33\%) atendem à grande maioria das especificações, entre elas às exigidas pelo setor aeroespacial. À medida que o teor de AN aumenta, a borracha nitrílica torna-se mais plástica e menos compatível com plastificantes ${ }^{[1,2]}$.

Fica claro, então, que o desenvolvimento de metodologias rápidas, de baixo custo, que indiquem, com precisão,

Autor para correspondência: Rita C. L. Dutra, Divisão de Química, Instituto de Aeronáutica e Espaço, AQI, CTA, CEP: 12 228-904, São José Campos, SP, Brasil. E-mail: chefia.aqi@iae.cta.br 
o teor de AN em NBR, é atrativo e importante para diversos setores da indústria de elastômeros, incluindo setores de ponta, como o aeroespacial ${ }^{[3]}$.

Também é conhecido, que a espectroscopia no infravermelho (IR) pode ser usada para identificar, caracterizar e quantificar elastômeros e suas misturas ${ }^{[4-7]}$.

A técnica de transmissão/pirólise sem controle de temperatura, ou seja, por bico de Bunsen, tem sido usada nos laboratórios da AQI/IAE para identificação de borrachas, ensaio acreditado pelo INMETRO. Outros trabalhos têm sido realizados em nossos laboratórios, empregando-se a pirólise controlada para diferentes tipos de materiais, incluindo elastômeros, de modo qualitativo ${ }^{[8,9]}$ e quantitativo ${ }^{[10]}$, com bons resultados.

Atualmente, novas adaptações em pirolisadores (Figura 1) têm sido propostas por Diniz, um dos autores, em nossos laboratórios (IAE/AQI), visando aumentar a quantidade de pirolisado para análise por FT-IR de materiais. Deste modo, um pirolisador foi idealizado, fabricado pela empresa Láctea, para aplicação em estudos quantitativos e usado, neste trabalho, para elastômeros.

Outra técnica FT-IR, de última geração, UATR, também está sendo usada, atualmente, nos laboratórios da AQI/IAE, para diferentes materiais, apresentando resultados quantitativos, com boa precisão ${ }^{[11,12]}$.

A Figura 2 mostra o acessório UATR contendo ZnSe e diamante, utilizado neste trabalho. $\mathrm{O}$ alto índice de refração do elemento ATR é adequado para obtenção de espectros de excelente qualidade para a maior parte das substâncias, com grau de penetração da radiação na amostra em torno de 1-2 $\mu \mathrm{m}$. A dureza e a estabilidade química do diamante permite a obtenção direta de espectros de materiais, com bom contato entre a amostra e o cristal ${ }^{[13]}$.

Borrachas são facilmente analisadas por técnicas de reflexão similares. A força do probe de análise pode ser ajustada a fim de se obter o contato mais adequado, uma vez que diferentes níveis de pressão influenciam diretamente as intensidades do espectro ATR obtido, o que impacta diretamente em resultados quantitativos ${ }^{[13]}$.

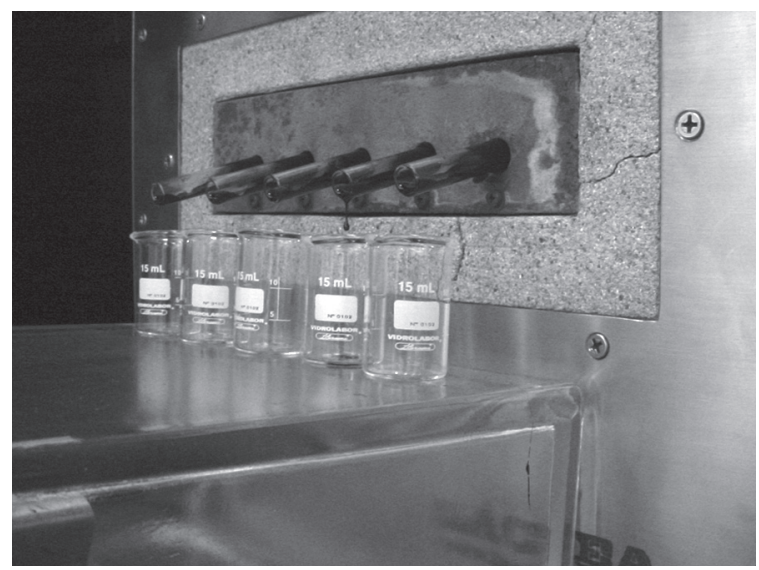

Figura 1. Pirolisador usado nesse trabalho/recolhimento dos pirolisados.

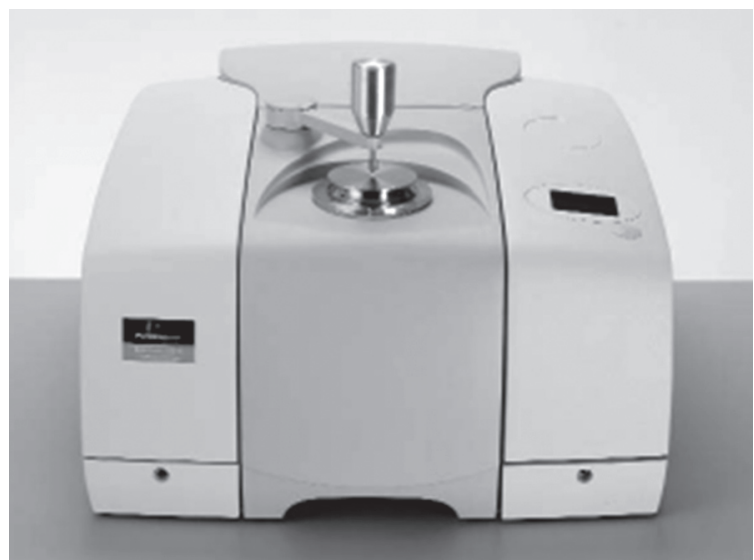

Figura 2. Acessório UATR usado nesse trabalho.

Mediante o exposto e com base nas características das técnicas FT-IR de reflexão (UATR) e transmissão (pirólise controlada), ambas disponíveis nos laboratórios da AQI/IAE, resolveu-se aplicá-las à determinação de AN em NBR, comparando-se dados como: tempo de análise e precisão.

\section{Experimental}

Amostras cruas de NBR, gentilmente, cedidas pela empresa Nitriflex, com diferentes teores de AN (N 726-28\%, $\mathrm{N} 7-30 \%, \mathrm{~N} 608-33 \%$, N 300-39\% e N 206-46\%) foram utilizadas para preparar, nos laboratórios da AQI/IAE, amostras vulcanizadas, conforme a formulação (phr e \%) mostrada na Tabela 1. Após mistura em misturador de rolos, foram moldadas sob pressão em placa de espessura $2 \mathrm{~mm}$ a $155^{\circ} \mathrm{C}$ por 15 minutos, tempo ótimo fornecido pelo reograma da mistura.

$\mathrm{Na}$ análise por FT-IR (técnica pirólise em bico de Bunsen), as amostras de borracha foram picadas e extraídas por 8 horas em extrator de Soxhlet, utilizando-se metanol como solvente. Após evaporação do solvente em estufa, foi colocada uma quantidade fixa de $0,3 \mathrm{~g}$ de amostra em cada tubo de pirólise. Foram feitas cinco alíquotas para cada amostra. Os tubos foram então levados para a chama do bico de Bunsen, colocados sempre numa mesma altura da chama (marcada com a ajuda de um suporte universal e uma garra para determinar a mesma posição do tudo a cada pirólise). Desde que a característica da técnica pirólise em bico de Bunsen é não haver controle de temperatura, foi feita uma tentativa de avaliar a

Tabela 1. Formulação das amostras de NBR com diferentes teores de AN.

\begin{tabular}{ccc}
\hline Ingrediente & phr & \% \\
\hline NBR & 100 & 74,6 \\
ZnO & 5 & 3,74 \\
Ac. Esteárico & 1 & 0,75 \\
Caolim & 20 & 14,9 \\
DOP & 5 & 3,73 \\
TMTD & 1 & 0,75 \\
MBTS & 1,5 & 1,12 \\
S & 0,5 & 0,37 \\
\hline
\end{tabular}


temperatura utilizada nos ensaios, com termopar e mantendo-se a capela ligada. Foram feitas 5 medidas de temperatura no interior do tubo de pirólise, durante o aquecimento em bico de Bunsen. Estes ensaios foram realizados utilizandose: 2 termopares tipo $\mathrm{K}$ com isolação mineral de $1,5 \mathrm{~mm}$ de diâmetro; Sistema 5000, Vishay Measurement Group, Inc; Scanner modelo 5100, sn: 125029; Cartão de leitura de temperatura, modelo 5120, sn: 126155; Cartão de interface, modelo PC5101A, sn: 149393; Notebook Tochiba Satellite, sn: X3151156P; Software Strainsment - Versão 3.1.14.490 Vishay Measurement Group, Inc.

Com 15 segundos de chama, começam a sair os primeiros vapores e inicia-se a condensação dos vapores na parte fria do tubo. Foi colocado um vidro de relógio pouco abaixo da extremidade do tubo de pirólise para que fosse recolhido o pirolisado. Com 40 segundos de queima a pirólise está completa. Com o auxílio de uma espátula, algumas gotas do pirolisado foram transferidas para a célula de $\mathrm{KBr}$ e analisadas, imediatamente, em espectrômetro FT-IR SPECTRUM ONE PERKIN ELMER (resolução $4 \mathrm{~cm}^{-1}$, ganho 1, $4000 \mathrm{a} 400 \mathrm{~cm}^{-1}, 40$ varreduras) como filmes líquidos com espaçador de $0,025 \mathrm{~mm}$.

Para a análise por FT-IR (técnica pirólise controlada), as amostras de borracha foram picadas e extraídas por 8 horas em metanol. Após evaporação do solvente, em estufa, foi colocada uma quantidade fixa de $0,3 \mathrm{~g}$ de amostra em cada tubo de pirólise.

Foram analisadas cinco alíquotas de cada amostra. Os tubos foram inseridos nos orifícios do pirolisador (já mostrados na Figura 1) na temperatura ambiente (somente até a profundidade onde estava a amostra). O pirolisador, foi então ligado, permitindo o aquecimento e quando atingiu a temperatura de $500^{\circ} \mathrm{C}$, os tubos foram introduzidos até o limite para que a borracha fosse toda pirolisada. Durante o aquecimento, ocorre a expansão dos gases liberados e a amostra tende a ser impulsionada para fora do tubo. O procedimento descrito para introdução do tubo foi feito com o objetivo de minimizar este efeito e garantir a pirólise completa de toda a amostra.

Pequenos beckers foram colocados próximos às bocas de cada tubo para recolher as gotas do pirolisado, conforme já mostrado na Figura 1.

Ao atingir a temperatura de $550{ }^{\circ} \mathrm{C}$, há uma boa estabilização de temperatura com uma precisão excelente em torno de $0,5^{\circ} \mathrm{C}$. As amostras foram mantidas nesta temperatura por 10 minutos, quando então o pirolisador foi desligado.

Os tubos foram retirados e os seus pirolisados foram analisados, imediatamente, em espectrômetro FT-IR SPECTRUM ONE PERKIN ELMER (resolução $4 \mathrm{~cm}^{-1}$, ganho 1, 4000 a $400 \mathrm{~cm}^{-1}, 40$ varreduras) como filmes líquidos com espaçador de $0,025 \mathrm{~mm}$.

Na técnica UATR, as amostras de borracha foram cortadas ao meio, de modo que a somente a parte interna do material ficasse em contato com o cristal, visando a análise específica do interior da amostra, sem interferência de espécies de superfície. Foram então analisadas, sem extração, cinco alíquotas de cada amostra. As amostras cortadas (em torno de $5 \mathrm{~mm}$ ) foram, simplesmente, colocadas em contato com a superfície do cristal de $\mathrm{ZnSe}$ com diamante, e foi aplicado um torque de $100 \mathrm{~N}$ com o braço articulado.

\section{Resultados e Discussão}

\section{Análise FT-IR}

\section{Técnica de transmissão/pirólise controlada}

A Figura 3 inclui os espectros FT-IR de transmissão (pirólise controlada) de algumas amostras de NBR estudadas. A linha de base para a banda $2237 \mathrm{~cm}^{-1}\left(\mathrm{~A}_{1}\right)$, atribuída ao grupo nitrila $\mathrm{C} \equiv \mathrm{N}$ de $\mathrm{AN}$ foi traçada de 2314 a $2112 \mathrm{~cm}^{-1}$ e para a banda de referência escolhida, $1455 \mathrm{~cm}^{-1}\left(\mathrm{~A}_{2}\right)$, atribuída aos grupos $\mathrm{CH}_{2}$ e $\mathrm{CH}_{3}$, de 1810 a $671 \mathrm{~cm}^{-1}$.

Pode-se observar que as intensidades das bandas estão relacionadas ao teor de AN em NBR, como esperado pela Lei de Lambert-Beer ${ }^{[14]}$. Com base nesta lei, e em concordância com o observado por Gedeon e colaboradores ${ }^{[5]}$, um entendimento das limitações do uso de pirólise IR para análise quantitativa deve ser feito. Para aplicar a lei de de LambertBeer a esta análise, os dados devem ser colocados em função da porcentagem da composição, Então, valores medianos de absorvância relativa $\mathrm{A}_{2237} / \mathrm{A}_{1455}\left(\mathrm{~A}_{1} / \mathrm{A}_{2}\right)$ de cinco (5) análises, foram calculados e colocados em função do teor de AN em NBR, para contornar problemas de variação de espessura.

A Tabela 2 apresenta os valores de $\left(\mathrm{A}_{1} / \mathrm{A}_{2}\right)$ (técnica: de transmissão/pirolise controlada) em função dos dados relativos ao teor de AN em NBR. Os valores de desvio médio padrão e relativo foram calculados de acordo com Hórak ${ }^{[15]}$, forma adotada em todos os trabalhos anteriores do grupo ${ }^{[7,10-12]}$ contendo análises quantitativas IR.

O erro relativo encontrado na metodologia pirólise controlada (1\%) está dentro dos limites de precisão do espectrômetro FT-IR, $\leq 2 \%$.

A Figura 4 mostra a curva analítica (técnica de transmissão/pirólise controlada). $\left(\mathrm{A}_{2237} / \mathrm{A}_{1455}\right) v s$. teor de $\mathrm{AN}(\% \mathrm{p} / \mathrm{p})$

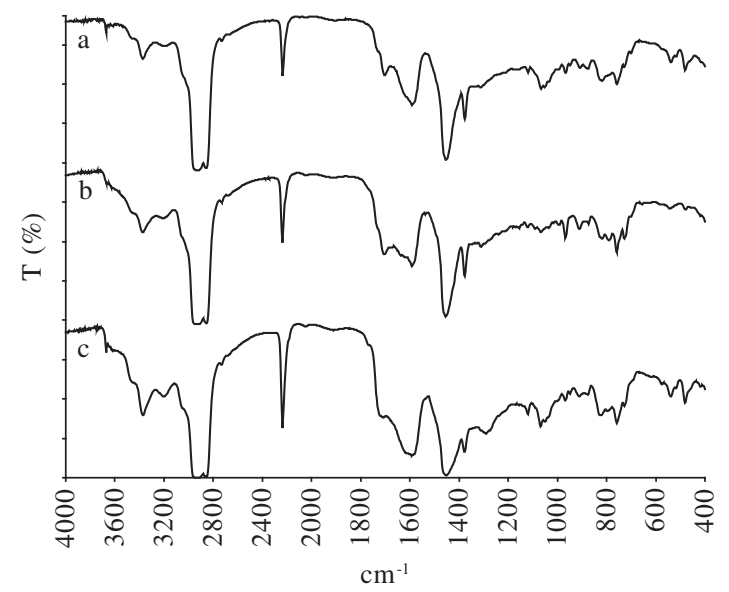

Figura 3. Espectros FT-IR (técnica de transmissão/pirólise controlada) de algumas amostras de NBR estudadas: a) $28 \% \mathrm{AN}$; b) $33 \%$ AN; e c) $46 \% \mathrm{AN}$. 
Sanches, N. B. et al. - Técnicas FT-IR de reflexão e de transmissão

Tabela 2. Dados FT-IR (técnica de transmissão/pirólise controlada) de borrachas NBR com diferentes teores de AN.

\begin{tabular}{|c|c|c|c|c|c|c|}
\hline Amostra & Banda analítica $2237 \mathrm{~cm}^{-1}$ & Banda referência $1455 \mathrm{~cm}^{-1}$ & $\mathbf{A}_{2237 / 1455}$ & Mediana & Desvio médio padrão & Desvio relativo (\%) \\
\hline \multirow{5}{*}{$\begin{array}{l}\text { N } 726 \\
(28 \%)\end{array}$} & 0,227 & 1,222 & 0,185 & \multirow{5}{*}{0,178} & \multirow{5}{*}{0,002} & \multirow{5}{*}{1,29} \\
\hline & 0,183 & 0,030 & 0,178 & & & \\
\hline & 0,203 & 1,139 & 0,178 & & & \\
\hline & 0,228 & 1,290 & 0,177 & & & \\
\hline & 0,205 & 1,184 & 0,173 & & & \\
\hline \multirow{5}{*}{$\begin{array}{c}\text { N } 7 \\
(30 \%)\end{array}$} & 0,242 & 1,273 & 0,190 & \multirow{5}{*}{0,192} & \multirow{5}{*}{0,001} & \multirow{5}{*}{0,40} \\
\hline & 0,250 & 1,289 & 0,194 & & & \\
\hline & 0,238 & 1,241 & 0,192 & & & \\
\hline & 0,221 & 1,153 & 0,191 & & & \\
\hline & 0,258 & 1,338 & 0,193 & & & \\
\hline \multirow{5}{*}{$\begin{array}{l}\text { N } 608 \\
(33 \%)\end{array}$} & 0,257 & 1,127 & 0,203 & \multirow{5}{*}{0,203} & \multirow{5}{*}{0,004} & \multirow{5}{*}{2,08} \\
\hline & 0,303 & 1,527 & 0,198 & & & \\
\hline & 0,408 & 2,005 & 0,203 & & & \\
\hline & 0,407 & 2,077 & 0,196 & & & \\
\hline & 0,353 & 1,621 & 0,218 & & & \\
\hline \multirow{5}{*}{$\begin{array}{l}\text { N 300 } \\
(39 \%)\end{array}$} & 0,340 & 1,479 & 0,230 & \multirow{5}{*}{0,239} & \multirow{5}{*}{0,002} & \multirow{5}{*}{0,80} \\
\hline & 0,278 & 1,163 & 0,239 & & & \\
\hline & 0,376 & 1,635 & 0,230 & & & \\
\hline & 0,284 & 1,183 & 0,240 & & & \\
\hline & 0,303 & 1,264 & 0,240 & & & \\
\hline \multirow{5}{*}{$\begin{array}{l}\text { N } 206 \\
(46 \%)\end{array}$} & 0,394 & 1,488 & 0,265 & \multirow{5}{*}{0,265} & \multirow{5}{*}{0,003} & \multirow{5}{*}{1,09} \\
\hline & 0,500 & 1,836 & 0,272 & & & \\
\hline & 0,424 & 1,635 & 0,259 & & & \\
\hline & 0,511 & 1,986 & 0,257 & & & \\
\hline & 0,472 & 1,781 & 0,265 & & & \\
\hline
\end{tabular}

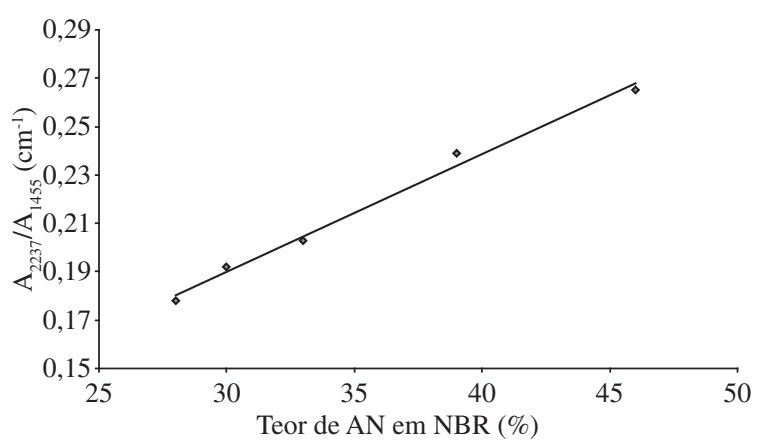

Figura 4. Curva analítica FT-IR (técnica de transmissão/pirólise controlada): $\left(\mathrm{A}_{2237} / \mathrm{A}_{1455}\right)$ vs. teores nominais de $\mathrm{AN}$.

em NBR. Da curva analítica (Tabela 2), a seguinte correlação $(\mathrm{R}=0,995)$ é proposta:

$$
Y=0,0049 x+0,0444
$$

onde $\mathrm{y}=$ valor mediano de $\mathrm{A}_{2237} / \mathrm{A}_{1455}$ e $\mathrm{X}$, o teor nominal de $\mathrm{AN}(\% \mathrm{p} / \mathrm{p})$ em NBR.

\section{Pirólise em bico de Bunsen}

Mesmo a técnica tendo como característica, a falta de controle de temperatura, foi feita uma tentativa para estimar a temperatura utilizada nos ensaios, conforme descrito no experimental. Foram realizados 5 ensaios e cada ensaio gerou aproximadamente 2400 pontos de temperatura, foram adotadas para se calcular a média, apenas o valor de temperatura em 40 segundos de queima, pois este tempo representa o final da pirólise, os valores obtidos foram: 541, 615, 604, 597 e $592{ }^{\circ} \mathrm{C}$, sendo a média dos valores, $590^{\circ} \mathrm{C}$.

A Figura 5 inclui os espectros FT-IR de transmissão (pirólise em bico de Bunsen) de algumas amostras de NBR estudadas. A linhas de base para as bandas $2237 \mathrm{~cm}^{-1}\left(\mathrm{~A}_{1}\right) \mathrm{e}$ $1455 \mathrm{~cm}^{-1}\left(\mathrm{~A}_{2}\right)$ foram as mesmas usadas na técnica pirólise controlada.

Pode-se observar, que do mesmo modo que a técnica de transmissão/pirólise controlada, as intensidades das bandas estão relacionadas ao teor de AN em NBR, como esperado pela Lei de Lambert-Beer ${ }^{[14]}$. O mesmo tipo de cálculo usado para a técnica de transmissão/pirólise controlada, foi feito.

A Tabela 3 apresenta os valores de $\left(\mathrm{A}_{1} / \mathrm{A}_{2}\right)$ (técnica de transmissão/pirolise em bico de Bunsen) em função dos dados relativos ao teor de AN em NBR. Os valores de desvio médio padrão e relativo foram calculados de acordo com Hórak $^{[15]}$, forma adotada em todos os trabalhos anteriores do grupo $^{[7,10-12]}$ contendo análises quantitativas IR.

O erro relativo encontrado na metodologia, usandose a técnica de transmissão/pirólise em bico de Bunsen (1\%) está dentro dos limites de precisão do espectrômetro FT-IR, $\leq 2 \%$. 


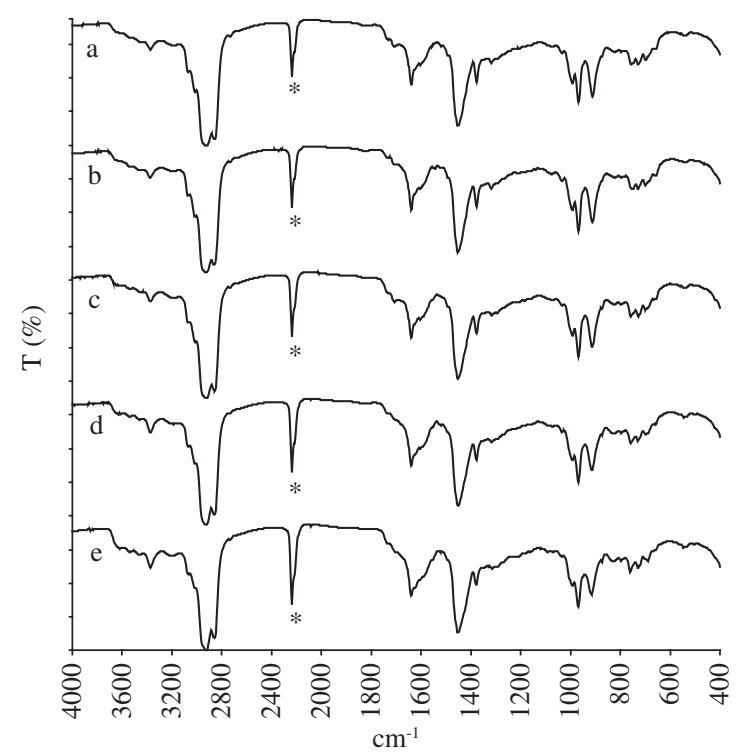

Figura 5. Espectros FT-IR (técnica de transmissão/pirólise em bico de Bunsen) de algumas amostras de NBR estudadas: a) $28 \% \mathrm{AN}$; b) $30 \% \mathrm{AN}$; c) $33 \% \mathrm{AN}$; d) $39 \% \mathrm{AN}$; e e) $46 \% \mathrm{AN}$.

A Figura 6 mostra a curva analítica (técnica de transmissão/pirólise controlada). $\left(\mathrm{A}_{2237} / \mathrm{A}_{1455}\right)$ vs. teor de $\mathrm{AN}(\% \mathrm{p} / \mathrm{p})$ em NBR. Da curva analítica (Tabela 3), a seguinte correlação $(\mathrm{R}=0 \ldots .$.$) é proposta:$

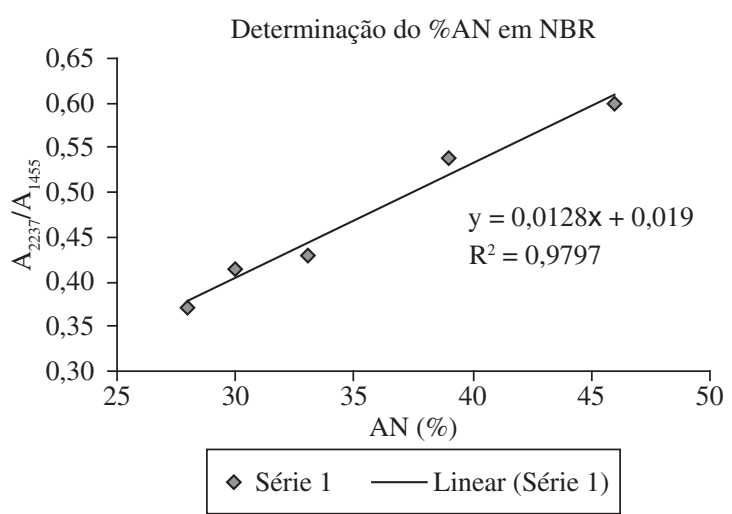

Figura 6. Curva analítica FT-IR (técnica pirólise em bico de Bunsen): $\left(\mathrm{A}_{2237} / \mathrm{A}_{1455}\right) v s$. teores nominais de $\mathrm{AN}$.

$$
Y=0,0128 x+0,019
$$

onde $\mathrm{y}=$ valor mediano de $\mathrm{A}_{2237} / \mathrm{A}_{1455}$ e $\mathrm{x}$, o teor nominal de AN (\%p/p) em NBR.

\section{Análise por reflexão/UATR}

A Figura 7 inclui os espectros de reflexão/UATR de algumas amostras de NBR estudadas. Pode-se observar que além da intensidade da banda em $2237 \mathrm{~cm}^{-1}$ estar relacionada ao teor de AN em NBR, como esperado pela Lei de Lambert-Beer ${ }^{[15]}$, nota-se ainda, no aspecto qualitativo, que a banda do polímero

Tabela 3. Dados FT-IR (técnica de transmissão /pirólise em bico de Bunsen) de borrachas NBR com diferentes teores de AN.

\begin{tabular}{|c|c|c|c|c|c|c|}
\hline Amostra & Banda analítica $2237 \mathrm{~cm}^{-1}$ & Banda referência $1455 \mathrm{~cm}^{-1}$ & $\mathbf{A}_{2237 / 1455}$ & Mediana & Desvio médio padrão & Desvio relativo $(\%)$ \\
\hline N 726 & 0,269 & 0,706 & 0,381 & & & \\
\hline \multirow[t]{4}{*}{$(28 \%)$} & 0,257 & 0,690 & 0,372 & & & \\
\hline & 0,252 & 0,681 & 0,370 & 0,372 & 0,007 & 1,88 \\
\hline & 0,308 & 0,758 & 0,406 & & & \\
\hline & 0,257 & 0,695 & 0,370 & & & \\
\hline N 7 & 0,296 & 0,701 & 0,422 & & & \\
\hline \multirow[t]{4}{*}{$(30 \%)$} & 0,284 & 0,687 & 0,413 & & & \\
\hline & 0,300 & 0,718 & 0,417 & 0,413 & 0,002 & 0,48 \\
\hline & 0,283 & 0,691 & 0,409 & & & \\
\hline & 0,286 & 0,695 & 0,411 & & & \\
\hline N 608 & 0,309 & 0,702 & 0,440 & & & \\
\hline \multirow[t]{4}{*}{$(33 \%)$} & 0,305 & 0,710 & 0,429 & & & \\
\hline & 0,299 & 0,711 & 0,420 & 0,429 & 0,004 & 0,93 \\
\hline & 0,301 & 0,699 & 0,431 & & & \\
\hline & 0,304 & 0,721 & 0,422 & & & \\
\hline N 300 & 0,371 & 0,690 & 0,538 & & & \\
\hline \multirow[t]{4}{*}{$(39 \%)$} & 0,351 & 0,655 & 0,536 & & & \\
\hline & 0,364 & 0,676 & 0,538 & 0,538 & 0,002 & 0,37 \\
\hline & 0,367 & 0,689 & 0,533 & & & \\
\hline & 0,366 & 0,673 & 0,544 & & & \\
\hline N 206 & 0,404 & 0,701 & 0,576 & & & \\
\hline \multirow[t]{4}{*}{$(46 \%)$} & 0,444 & 0,724 & 0,613 & & & \\
\hline & 0,413 & 0,675 & 0,611 & 0,600 & 0,007 & 1,17 \\
\hline & 0,427 & 0,713 & 0,599 & & & \\
\hline & 0,424 & 0,707 & 0,600 & & & \\
\hline
\end{tabular}




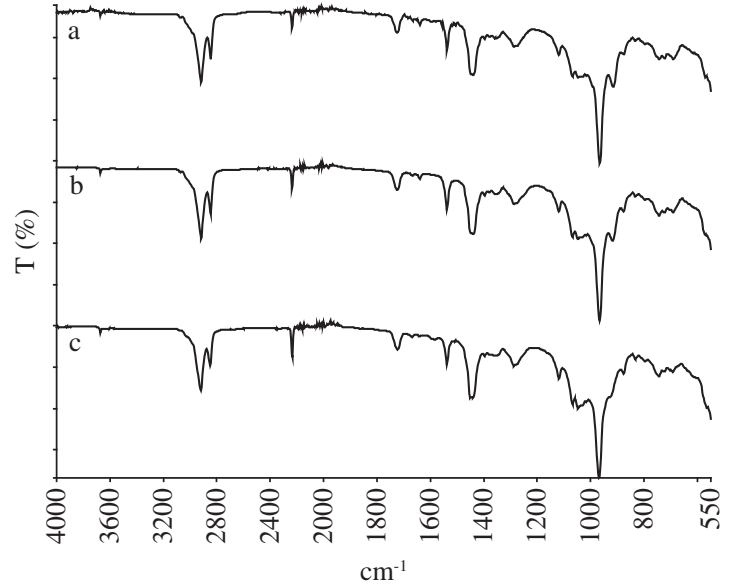

Figura 7. Espectros FT-IR (técnica de reflexão/UATR) de algumas amostras de NBR estudadas: a) $28 \% \mathrm{AN}$; b) $33 \% \mathrm{AN}$; e c) $46 \% \mathrm{AN}$.

NBR dos grupos $\mathrm{C}=\mathrm{C}$ trans, em $970 \mathrm{~cm}^{-1}$, é bem evidenciada, além de pequena absorção em $1725 \mathrm{~cm}^{-1}$, que pode ser atribuída ao grupo $\mathrm{C}=\mathrm{O}$ de algum aditivo carbonilado. Essas diferenças em relação aos espectros de NBR obtidos pela técnica pirólise controlada podem ser relacionadas com as características das técnicas. A pirólise é uma degradação térmica e no caso de elastômeros ${ }^{[4,8]}$, as absorções do pirolisado, após extração, estão associadas com o respectivo monômero. No caso do método de reflexão/UATR, as absorções do polímero e de espécies de superfície, que podem ser aditivos, são visualizadas.

A Tabela 4 apresenta os valores de $\left(\mathrm{A}_{1}\right)$ (método de reflexão/UATR) em função dos dados relativos ao teor de AN em NBR. O erro relativo encontrado na metodologia pirólise controlada (1\%) está dentro dos limites de precisão do espectrômetro FT-IR, $\leq 2 \%$.

A Figura 8 mostra a curva analítica UATR $\left(\mathrm{A}_{2237}\right)$ vs. teor de AN (\%p/p) em NBR. Da curva analítica (Tabela 3), a seguinte correlação $(R=0,996)$ é proposta:

$$
\mathrm{Y}=0,0014 \mathrm{x}-0,002
$$

onde $\mathrm{y}=$ valor mediano de $\mathrm{A}_{2237}$ e $\mathrm{x}$, o teor nominal de $\mathrm{AN}(\% \mathrm{p} / \mathrm{p})$ em NBR.

Considerando-se dois fatores: 1) a mistura de duas borrachas NBR com diferentes teores de AN fornece borracha nitrílica com valor intermediário de acrilonitrila ${ }^{[1,2]}$; e 2) o tempo gasto para análise, usando-se a técnica de transmissão/pirólise em bico de Bunsen é menor que o da técnica de transmissão/pirolise controlada, decidiu-se manufaturar, em nossos laboratórios, misturas de borrachas NBR, de teor de AN conhecido, usá-las para teste das curvas e analisá-las por meio da técnica de transmissão/pirólise em bico de Bunsen em comparação à de reflexão/UATR. A precisão dos dados obtidos das analises FT-IR por ambos procedimentos (transmissão/pirólise em bico de Bunsen e reflexão/UATR) é mostrada na Tabela 5. Os resultados foram considerados bons, havendo concordância entre as técnicas.

Embora as técnicas de reflexão/UATR e de transmissão, usando pirólise controlada ou em bico de Bunsen para pre-
Tabela 4. Dados FT-IR (reflexão/UATR) de borrachas NBR com diferentes teores de AN.

\begin{tabular}{|c|c|c|c|c|}
\hline Amostra & $\begin{array}{c}\text { Banda analítica } \\
2237\left(\mathrm{~cm}^{-1}\right)\end{array}$ & Mediana & $\begin{array}{l}\text { Desvio } \\
\text { padrão }\end{array}$ & $\begin{array}{c}\text { Desvio } \\
\text { relativo (\%) }\end{array}$ \\
\hline \multirow{5}{*}{$\begin{array}{c}\text { N } 726 \\
28 \%\end{array}$} & 0,038 & & & \\
\hline & 0,038 & & & \\
\hline & 0,039 & 0,039 & 0,000 & 0,99 \\
\hline & 0,039 & & & \\
\hline & 0,040 & & & \\
\hline \multirow{5}{*}{$\begin{array}{l}\text { N } 7 \\
30 \%\end{array}$} & 0,040 & & & \\
\hline & 0,040 & & & \\
\hline & 0,041 & 0,041 & 0,000 & 0,47 \\
\hline & 0,041 & & & \\
\hline & 0,041 & & & \\
\hline \multirow{5}{*}{$\begin{array}{c}\text { N } 608 \\
33 \%\end{array}$} & 0,042 & & & \\
\hline & 0,044 & & & \\
\hline & 0,045 & 0,045 & 0,001 & 1,71 \\
\hline & 0,046 & & & \\
\hline & 0,046 & & & \\
\hline \multirow{5}{*}{$\begin{array}{c}\text { N } 300 \\
39 \%\end{array}$} & 0,054 & & & \\
\hline & 0,055 & & & \\
\hline & 0,056 & 0,056 & 0,001 & 1,03 \\
\hline & 0,056 & & & \\
\hline & 0,057 & & & \\
\hline \multirow{5}{*}{$\begin{array}{c}\text { N } 206 \\
46 \%\end{array}$} & 0,062 & & & \\
\hline & 0,064 & & & \\
\hline & 0,064 & 0,064 & 0,001 & 0,90 \\
\hline & 0,065 & & & \\
\hline & 0,065 & & & \\
\hline
\end{tabular}

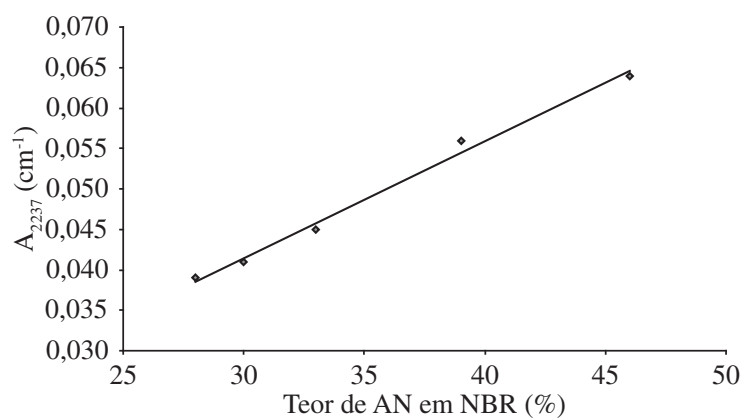

Figura 8. Curva analítica FT-IR (técnica de reflexão/UATR): $\left(\mathrm{A}_{2237}\right) v s$. teores nominais de $\mathrm{AN}$.

paração de amostras, apresentem precisão similar, o tempo para obtenção dos resultados (experimental e cálculo) é menor utilizando-se a técnica UATR (30 minutos) contra 1 hora 30 minutos, técnica pirólise controlada ou em bico de Bunsen, sem extração, para esse tipo determinação.

Apesar da banda analítica, $2237 \mathrm{~cm}^{-1}$ ser a mesma para as técnicas e estar em região intermediária do espectro FT-IR, livre de bandas vizinhas, e deste modo para a determinação não haveria necessidade de extração das amostras, no caso da pirólise controlada e em bico de Bunsen, essa etapa foi necessária pois foi usada banda de referência, situada na região 
Sanches, N. B. et al. - Técnicas FT-IR de reflexão e de transmissão

Tabela 5. Dados de precisão da metodologia FT-IR transmissão/(pirólise em bico de Bunsen e reflexão/UATR).

\begin{tabular}{|c|c|c|c|c|c|c|c|c|c|}
\hline Amostra & $\begin{array}{c}\text { Banda } \\
\text { analítica } \\
2237 \\
\left(\mathrm{~cm}^{-1}\right) \\
\text { (UATR) }\end{array}$ & $\begin{array}{c}\text { Valor } \\
\text { mediano- } \\
\text { banda } \\
\text { analítica } \\
2237\left(\mathbf{c m}^{-1}\right) \\
\text { (UATR) }\end{array}$ & $\begin{array}{c}\text { Banda relativa } \\
\mathbf{A}_{2237} / \mathbf{A}_{1455}\left(\mathrm{~cm}^{-1}\right) \\
\text { (pirólise em } \\
\text { bico de Bunsen) }\end{array}$ & $\begin{array}{c}\text { Valor } \\
\text { mediano-banda } \\
\text { relativa } \\
\mathbf{A}_{2237} / \mathbf{A}_{1455}\left(\mathrm{~cm}^{-1}\right) \\
(\text { pirólise } \mathrm{em} \\
\text { bico de Bunsen) } \\
\end{array}$ & $\begin{array}{c}\text { Desvio } \\
\text { relativo } \\
\text { (pirólise } \\
\text { em bico de } \\
\text { Bunsen) } \\
(\%)\end{array}$ & $\begin{array}{c}\text { Desvio } \\
\text { relativo } \\
\text { (UATR) } \\
(\%)\end{array}$ & $\begin{array}{c}\text { Teor } \\
\text { de AN } \\
\text { téorico } \\
(\%)\end{array}$ & $\begin{array}{c}\text { Concentração } \\
\text { de AN por } \\
\text { UATR } \\
(\% \mathbf{m} / \mathbf{m})\end{array}$ & $\begin{array}{c}\text { Concentração } \\
\text { de AN por } \\
\text { pirólise } \\
\text { em bico de } \\
\text { Bunsen } \\
(\% \mathrm{~m} / \mathrm{m}))\end{array}$ \\
\hline \multirow{5}{*}{$\begin{array}{c}\text { Mistura de } \\
\text { N 386B e } \\
\text { N } 300\end{array}$} & 0,054 & & 0,543 & & & & & & \\
\hline & 0,055 & & 0,527 & & & & & & \\
\hline & 0,054 & 0,054 & 0,534 & 0,529 & 0,57 & 0,74 & 40 & 40 & 39,8 \\
\hline & 0,055 & & 0,529 & & & & & & \\
\hline & 0,053 & & 0,528 & & & & & & \\
\hline \multirow{5}{*}{$\begin{array}{l}\text { Mistura de } \\
\quad \text { N } 7 \\
26+\text { N } 206\end{array}$} & 0,049 & & 0,485 & & & & & & \\
\hline & 0,049 & & 0,490 & & & & & & \\
\hline & 0,049 & 0,049 & 0,493 & 0,490 & 0,62 & 0 & 37 & 36,4 & 36,8 \\
\hline & 0,048 & & 0,486 & & & & & & \\
\hline & 0,049 & & 0,496 & & & & & & \\
\hline
\end{tabular}

de impressão digital, onde a interferência de aditivos é maior. O uso de extração torna bem mais expressiva a diferença entre as técnicas, com relação ao tempo de análise.

\section{Conclusão}

Foram desenvolvidos três métodos FT-IR, usando-se técnicas de reflexão (UATR), transmissão/pirólise em bico de Bunsen e transmissão/pirólise controlada, para determinar o teor de AN em formulações de NBR vulcanizadas, a partir de formulações onde a concentração de acrilonitrila seja conhecida.

Os métodos são puramente quantitativos dependentes de se ter disponível elastômeros NBR com concentração de AN conhecida para a construção da curva analítica de correlação, porém independe do tipo e teor de carga presente o que o torna uma ferramenta muito útil para controle de qualidade de processo ou recebimento de matéria prima.

Embora, as técnicas apresentem precisão similar, o tempo para obtenção dos resultados é menor utilizando-se a técnica UATR, além desta técnica de reflexão ser um ensaio não-destrutivo e requerer pequena quantidade de amostra, em torno de $5 \mathrm{~mm}$.

\section{Referências Bibliográficas}

1. Elastômeros nitrílicos, Nitriflex S.A. Ind e Com. (Catálogo Nitriflex) Rio de Janeiro (1984)

2. Iozzi, M. A; Martins, A. M. \& Mattoso, L. A. C. - Polímeros: Ciência e Tecnologia, 14, (2), p.92, (2004).

3. Holanda, J. A. S. - "A influência do tipo e teor de cargas nas propriedades mecânicas de elastômeros usados como isolantes térmicos em motores-foguetes", Tese de Mestrado, Instituto Tecnológico da Aeronáutica, São José dos Campos, Brasil (2004).

4. Wake, W. C.; Tidd, B. K. \& Loadman, M. J. R. - "Analysis of Rubber and Rubber-like Polymers", third Edition, Applied Science Publishers (1983).
5. Gedeon, B. J. \& Ngyuen, R. H. - Rubber Division Meeting, American Chemical Society, Cleveland, Ohio, Oct 14, paper 64 (1985).

6. Annual Book of ASTM Standards, Part 37 ASTM D3677 (1982).

7. Dutra, R. C. L. - Analyse quantitative infrarouge des associations du caoutchouc, IFOCA, Paris, (1988).

8. Sanches, N. B; Diniz, M. F.; Reis, T. B.; Cassu, S. N. \& Dutra, R. C. L. - Polímeros: Ciência e Tecnologia, 16, (3), p.211 (2006).

9. Romão, B. M. V.; Diniz, M. F; Azevedo, M. F. P; Lourenço, V. L.; Pardini, L. C. \& Dutra, R. C. L. - Polímeros: Ciência e Tecnologia, 16, (3), p.94 (2006).

10. Dutra, R. C. L.; Diniz, M. F.; Ribeiro, A. P.; Lourenço, V. L.; Cassu, S. N. \& Azevedo, M. F. P. - Polímeros: Ciência e Tecnologia, 14, (5), 334, (2004).

11. Siqueira, S. H. S.; Diniz, M. F. \& Dutra, R. C. L. - Polímeros: Ciência e Tecnologia, in press (2008).

12. Mattos, E. C. M. - "Caracterização e quantificação, por meio de técnicas FT-IR, HPLC e TG, de polímeros utilizados em composições de explosivos plásticos”, Tese de Doutorado, Instituto Tecnológico da Aeronáutica, São José dos Campos, Brasil (2007).

13. PERKIN ELMER; Technical Note - Improved Performance with the New Spectrum 100 UATR Accessory - www. perkinelmer.com.

14. Smith, A. L. - “Applied Infrared Spectroscopy”, John Wiley \& Sons, New York, (1979).

15. Hórak, M. \& Vítek, A. -"Interpretation and processing of Vibrational Spectra" John Wiley \& Sons, New York, (1978).

16. Dutra, R. C. L.; Lourenço, V. L.; Diniz, M. F.; Azevedo, M. F. P.; Barbosa, R. V. \& Soares, B. G. - Polymer Bulletin, 36, p.593 (1996).

Enviado: 21/12/07

Reenviado: 30/03/08

Aceito: 06/05/08 